LA W RENCE LIVERMORE N A TIO NAL LABORATORY
Wave-Based Subsurface Guide Star

S. K. Lehman

August 15, 2011 
This document was prepared as an account of work sponsored by an agency of the United States government. Neither the United States government nor Lawrence Livermore National Security, LLC, nor any of their employees makes any warranty, expressed or implied, or assumes any legal liability or responsibility for the accuracy, completeness, or usefulness of any information, apparatus, product, or process disclosed, or represents that its use would not infringe privately owned rights. Reference herein to any specific commercial product, process, or service by trade name, trademark, manufacturer, or otherwise does not necessarily constitute or imply its endorsement, recommendation, or favoring by the United States government or Lawrence Livermore National Security, LLC. The views and opinions of authors expressed herein do not necessarily state or reflect those of the United States government or Lawrence Livermore National Security, LLC, and shall not be used for advertising or product endorsement purposes.

This work performed under the auspices of the U.S. Department of Energy by Lawrence Livermore National Laboratory under Contract DE-AC52-07NA27344. 


\title{
Wave-Based Subsurface Guide Star
}

\author{
Sean K. Lehman
}

July 19, 2011

\begin{abstract}
Astronomical or optical guide stars are either natural or artificial point sources located above the Earth's atmosphere. When imaged from ground-based telescopes, they are distorted by atmospheric effects. Knowing the guide star is a point source, the atmospheric distortions may be estimated and, deconvolved or mitigated in subsequent imagery. Extending the guide star concept to wave-based measurement systems to include acoustic, seismo-acoustic, ultrasonic, and radar, a strong artificial scatterer (either acoustic or electromagnetic) may be buried or inserted, or a pre-existing or natural sub-surface point scatterer may be identified, imaged, and used as a guide star to determine properties of the sub-surface volume. That is, a data collection is performed on the guide star and the sub-surface environment reconstructed or imaged using an optimizer assuming the guide star is a point scatterer. The optimization parameters are the transceiver height and bulk sub-surface background refractive index. Once identified, the refractive index may be used in subsequent reconstructions of sub-surface measurements. The wave-base guide star description presented in this document is for a multimonostatic ground penetrating radar (GPR) but is applicable to acoustic, seismo-acoustic, and ultrasonic measurement systems operating in multimonostatic, multistatic, multibistatic, etc., modes.
\end{abstract}

\section{Introduction}

Most wave-based (acoustic, seismo-acoustic, ultrasonic, or radar) remote sensing and imaging systems require knowledge of the background environment and transceiver locations. In the case of a two layered medium, the required parameters are:

- The refractive index, $n_{1}$, of the "top" layer, that is the layer in which the transceivers exist. For most cases, $n_{1} \equiv 1$.

- The refractive index, $n_{2}$, of the "bottom" layer, that is the layer to be probed. This is unknown.

- The transducer positions,

- $\left\{\mathbf{R}_{n}^{\mathrm{src}} \equiv\left(x_{n}^{\mathrm{src}}, y_{n}^{\mathrm{src}}, z_{n}^{\mathrm{src}}\right)\right\}_{n=1}^{N_{\mathrm{src}}}$ for sources and $\left\{\mathbf{R}_{m}^{\mathrm{rcv}} \equiv\left(x_{m}^{\mathrm{rcv}}, y_{m}^{\mathrm{rcv}}, z_{m}^{\mathrm{rcv}}\right)\right\}_{m=1}^{N_{\mathrm{rcv}}}$ for receivers for multistatic systems;

- $\left\{\mathbf{R}_{n}^{\mathrm{xvr}} \equiv\left(x_{n}^{\mathrm{xvr}}, y_{n}^{\mathrm{xvr}}, z_{n}^{\mathrm{xvr}}\right)\right\}_{n=1}^{N_{\mathrm{rcv}}}$ for multimonostatic transceivers;

- $\left\{\mathbf{R}_{n}^{\mathrm{src}} \equiv\left(x_{n}^{\mathrm{src}}, y_{n}^{\mathrm{src}}, z_{n}^{\mathrm{src}}\right)\right\}_{n=1}^{N_{\mathrm{xvr}}}$ and $\left\{\mathbf{R}_{n}^{\mathrm{rcv}} \equiv\left(x_{n}^{\mathrm{rcv}}, y_{n}^{\mathrm{rcv}}, z_{n}^{\mathrm{rcv}}\right)\right\}_{n=1}^{N_{\mathrm{xvr}}}$ for multibistatic systems where either the receiver positions depend upon the source locations, or vice-versa;

must also be known ${ }^{1}$. Generally, the transducers' planar, $(x, y)$, locations are known from the positioning system. Their heights above the surface are not consistently known and must be determined.

A wave-based multilayer guide star procedure is proposed for deriving the unknown physical parameters required for imaging. A strong point scatterer is either inserted into, buried in, or an existing point scatterer identified in, the sub-surface volume ${ }^{2}$. The point scatterer is taken to be the guide star. Data are collected on it and the subsurface

\footnotetext{
${ }^{1}$ See Section 4 for a description of operating modes.

${ }^{2}$ When a guide star is buried or inserted into the medium, it is considered to be artificial. An identified, existing, guide star is known as natural.
} 


\begin{tabular}{|l|l|l|l|}
\hline $\begin{array}{l}\text { Subsurface } \\
\text { Material }\end{array}$ & $\begin{array}{l}\text { Guide Star } \\
\text { Object }\end{array}$ & $\begin{array}{l}\text { Resolution } \\
\text { Phantom }\end{array}$ & $\begin{array}{l}\text { Lathe } \\
\text { Phantom }\end{array}$ \\
\hline 1) Sand & & & \\
\hline 2) Gravel & & & \\
\hline 3) Dirt & & & \\
\hline
\end{tabular}

Table 1: GPR guide star test matrix. There are nine proposed data collections.

volume is imaged using an optimization algorithm using the assumption the guide start should reconstruct as a point scatterer. The optimized parameters include the bulk refractive index and height of the transducers.

A proof-of-principle is offered based upon a ground penetrating radar sub-surface measurement system but the concept is applicable to all wave-based measurement and imaging systems.

\section{GPR Proof-of-Principle}

A ground penetrating radar (GPR) guide star experiment and procedure is proposed for deriving the unknown physical parameters required for imaging. Figure 1 presents a multimonostatic data collection concept ${ }^{3}$. Figure 2 shows a detail of the geometry with the parameters to be derived: the sub-surface refractive index $n_{2}$ and the transceiver height, $z_{0}-z_{n}$.

The process consists of burying a highly scattering long object with cross-section much less than the shortest useable operating pulse wavelength, that is a "thin" object. This is the guide star. The object must be a thin pole or thick wire ${ }^{4}$. Data are collected over the guide star and the subsurface imaged using a model-based processor (MBP) which maximizes the scattering cross-section of the guide star by optimizing the unknown parameters under the assumption the guide star is the most highly scattering object in the environment.

An overly detailed description of a model-based processor (MBP) is presented in Figure 3. The salient feature for this discussion is the the forward physical propagation model, $\hat{h}\left(\mathbf{p}_{k}\right)$. As the MBP operates iteratively, a fast forward computation model is required. Thus, it is proposed to take advantage of recent advances in fast forward electromagnetic solvers in this work.

After the guide star optimizer has determined the bulk refractive index of the subsurface, two phantoms will be buried and data collected on them:

- The aluminum pole resolution phantom of Figure 4 , and

- The lathe phantom of Figure 5.

For the case of the lathe phantom, the optimizer will be modified to fit the lathe components: mean radius, length, and mean depth.

As there are sand, gravel, and dirt boxes in B181, it would be of additional interest to perform identical data collections in each of the materials. Thus, nine data collections are envisioned: the guide star followed by the two phantoms (three objects) successively buried in three differing materials. The test matrix is presented in Table 1 .

\section{Data Collection}

As this is a proof-of-principle experiment, it is desired to have the data collection aspects as rigidly controlled as possible. Thus, the sandboxes must be carefully prepared and groomed by loosening the material, and removing any foreign objects or inhomogeneities. The objects must be buried "flat" or parallel to the surface. The surface must be smoothed to be flat and parallel to the array.

\footnotetext{
${ }^{3}$ The guide star concept described herein applies equally to fully multistatic or multibistatic systems.

${ }^{4}$ The object must not be "too thin" as its backscattered field must be measurable.
} 


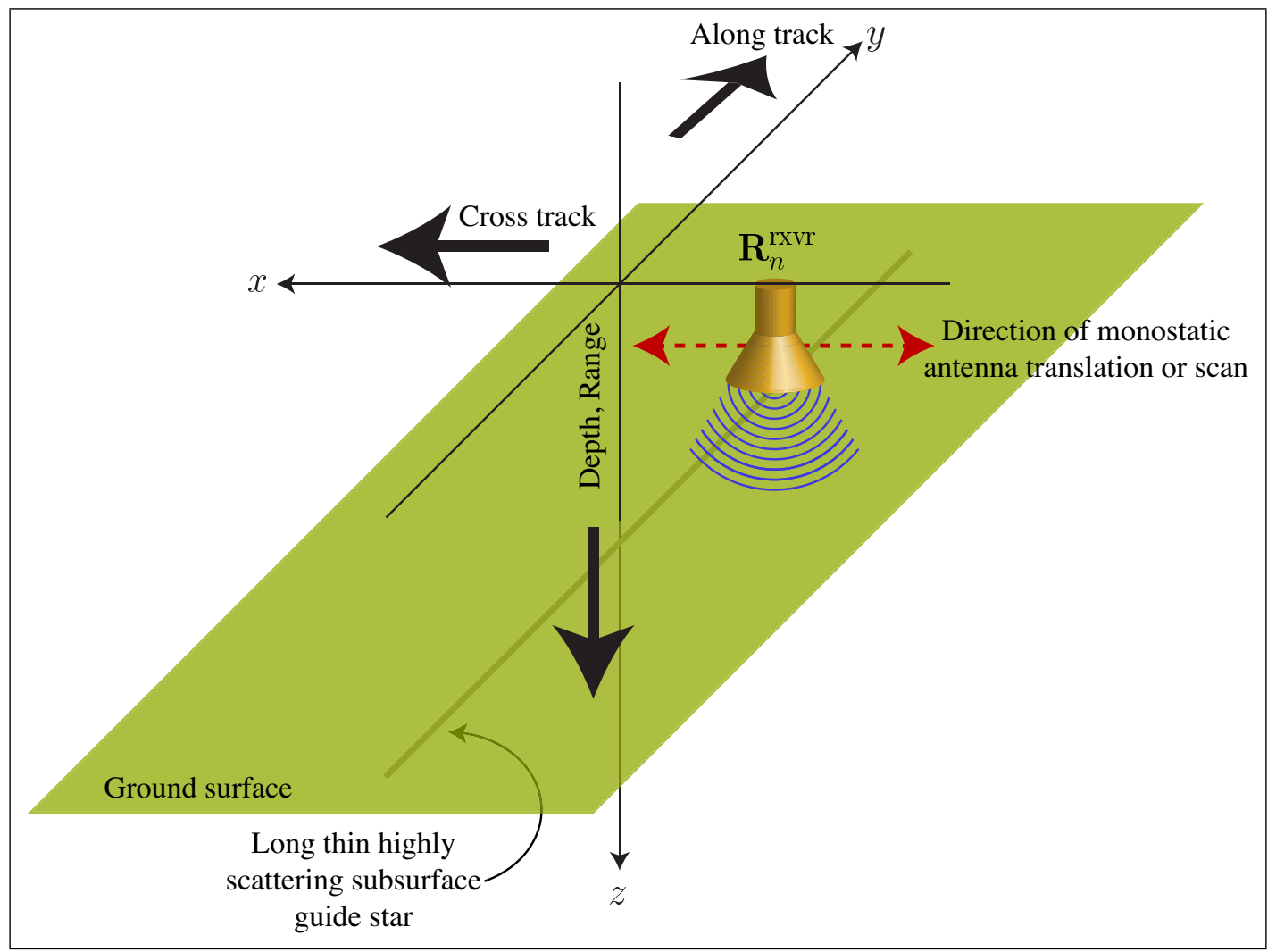

Figure 1: Multimonostatic ground penetrating radar (GPR) measurement geometry.

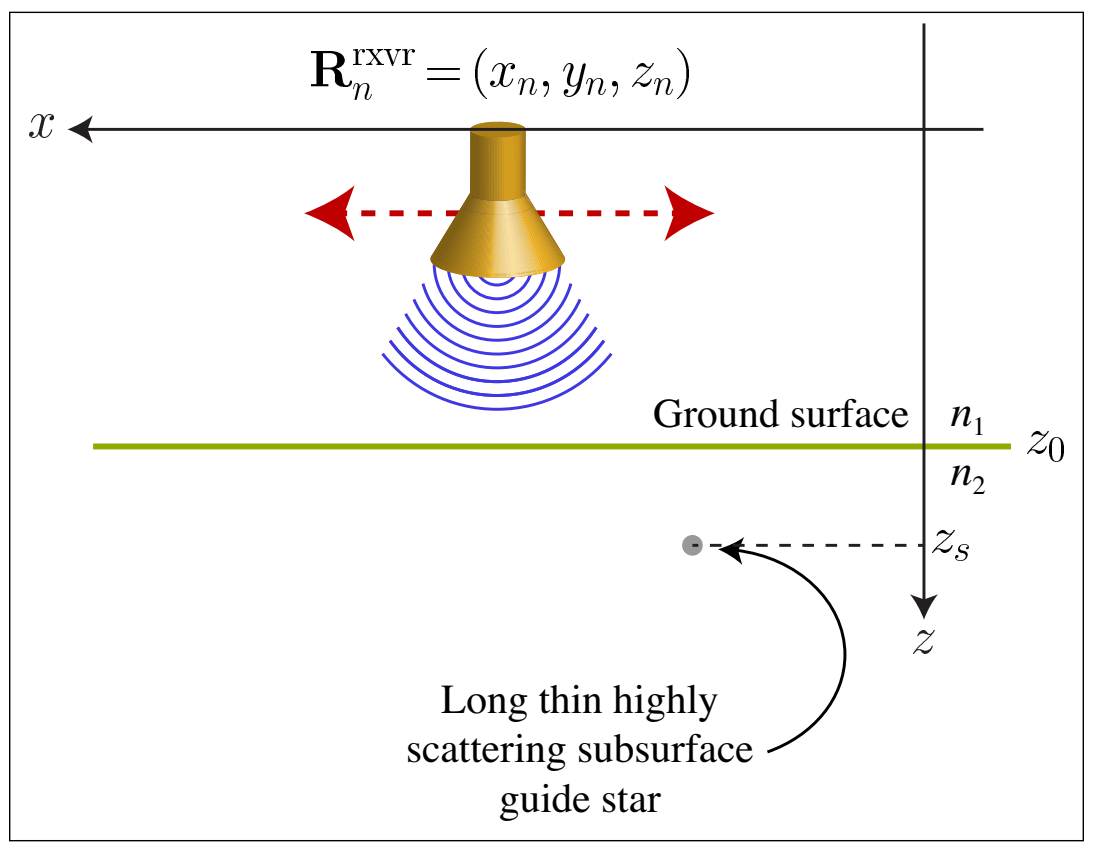

Figure 2: Detailed view of GPR measurement geometry. 


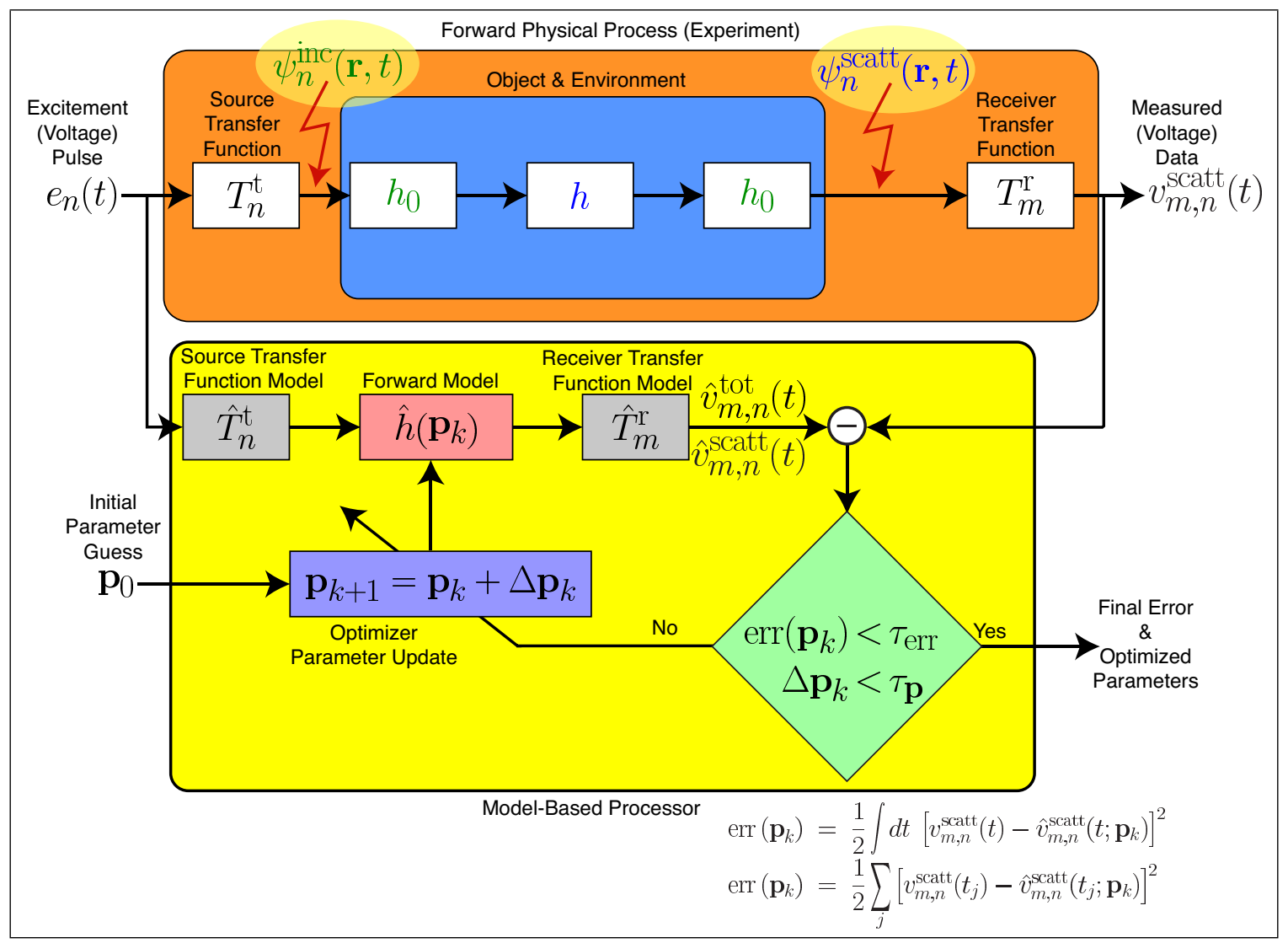

Figure 3: Over-detailed iterative model based processor. 


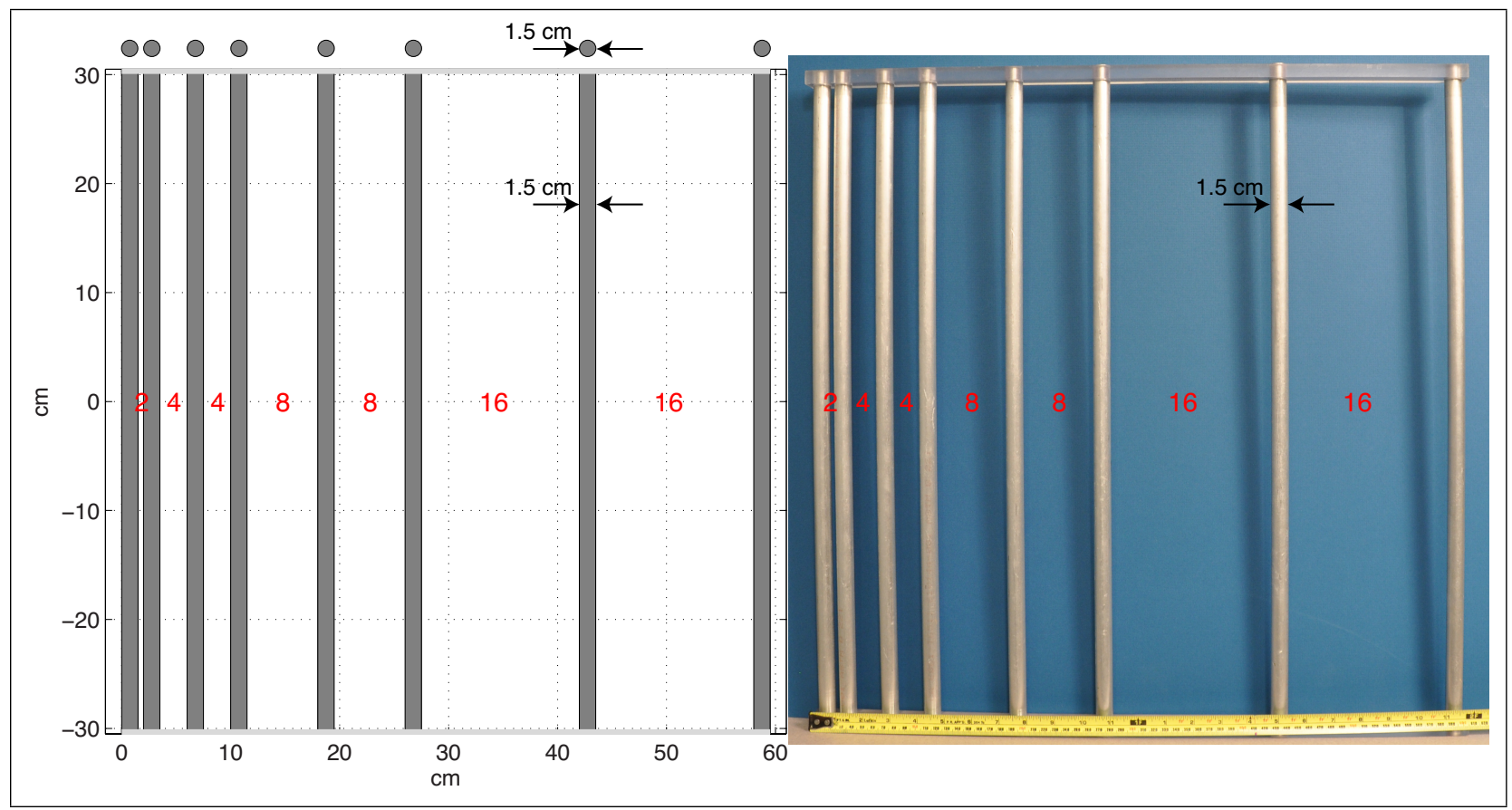

Figure 4: Aluminum pole resolution phantom.

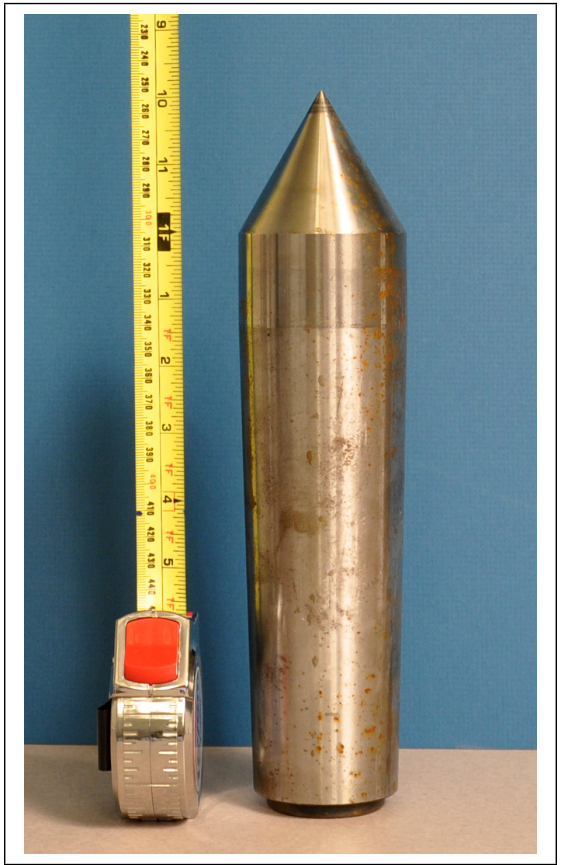

Figure 5: Lathe phantom. 


\section{Measurement System Operating Modes}

There are many measurement system operating modes or data collection methods. The common ones are

Monostatic : A transceiver remains fixed in space, $\mathbf{R}_{\mathrm{xvr}} \equiv$ constant. An incident field is emitted and any backscattered fields are measured. This is known as "pulse/echo" operation. The data are represented by $v^{\text {scat }}(t)$.

Multimonostatic : A monostatic transceiver performs pulse/echo measurements at successive spatial locations indicated by $\left\{\mathbf{R}_{n}^{\mathrm{xvr}}\right\}_{n=1}^{N_{\mathrm{xvr}}}$.

Bistatic : A source at a fixed location, $\mathbf{R}^{\text {src }} \equiv$ constant, emits an incident field. A spatially separated receiver at a fixed location, $\mathbf{R}^{\mathrm{rcv}} \equiv$ constant $\neq \mathbf{R}^{\mathrm{src}}$, measures any scattered fields. The incident field may also be measured. This is known as "pitch/catch" operation. The data are represented by $v^{\text {scat }}(t)$.

Multistatic : Successively, multiple spatially separated sources, located at $\left\{\mathbf{R}_{n}^{\mathrm{src}}\right\}_{n=1}^{N_{\text {src }}}$, emit incident fields. Scattered fields are measured simultaneously at multiple spatially separated receivers, located at $\left\{\mathbf{R}_{m}^{\mathrm{rcv}}\right\}_{m=1}^{N_{\mathrm{rcv}}}$. Sources and receivers may share common spatial locations. The data are represented by $v_{m n}^{\text {scat }}(t)$.

Multibistatic : A bistatic pair with a fixed offset perform pitch/catch operations at successive spatial locations, $\left\{\mathbf{R}_{n}^{\text {src }}, \mathbf{R}_{n}^{\text {rcv }}\right\}_{n=1}^{N_{\text {xvr }}}$ where $\left|\mathbf{R}_{n}^{\text {rcv }}-\mathbf{R}_{n}^{\text {src }}\right| \equiv$ constant. The data are represented by $v_{n}^{\text {scat }}(t)$.

Variable Offset Multibistatic : A multibistatic pair perform pitch/catch operations with one transceiver fixed while the other operates at successive spatial locations. The locations are described by one of

- $\mathbf{R}^{\mathrm{src}} \equiv$ constant with multiple receiver positions, $\left\{\mathbf{R}_{m}^{\mathrm{rcv}}\right\}_{m=1}^{N_{\mathrm{rcv}}}$, or

- $\mathbf{R}^{\mathrm{rcv}} \equiv$ constant with multiple source positions, $\left\{\mathbf{R}_{n}^{\mathrm{src}}\right\}_{n=1}^{N_{\text {src }}}$.

The data are represented by $v_{m n}^{\text {scat }}(t)$ where $m$ can be a function of $n$ or vice-versa. 